\title{
HPV Vaccine: Public Health, Politics, and Personal Choice
}

\author{
Sandra K. Cesario*
}

$\mathrm{H}$ uman papillomavirus (HPV) is known to be associated with many types of cancers disproportionately affecting women. The most common of these is cervical cancer. However anal, vulvar, vaginal, penile, and oropharyngeal cancers have also been linked to this virus. It is estimated that 527624 women receive a cervical cancer diagnosis annually and 265653 die from the disease making it the third most common cancer among women globally (1). Anal cancer, while not as prevalent as cervical cancer, is on the rise with approximately 27000 new cases identified worldwide each year. Women are at higher risk than men for anal cancer, especially those with cervical or vulvar cancers and those who are immunocompromised. Other conditions such as recurrent juvenile respiratory papillomatosis and genital warts are often attributed to HPV types 6 and 11.

\section{HPV and Cancer Etiology Worldwide}

HPV is sexually transmitted. Sexual activity that begins at an early age, lack of condom use, and engaging in sex with multiple partners are strong risk factors for the spread of this disease. There are approximately 180 different types of double-stranded DNA viruses known collectively as HPV that have been linked to both benign and malignant skin and squamous mucosae lesions. Some of these viruses which simply produce warts are viewed as low-risk (eg, HPV 6 and 11). Other types are considered to be high risk (eg, HPV 16, 18, 31, 33, 35, 39, 45, 51, 52, 56, and 58) and may result in tumor growth (2). Almost $100 \%$ of cervical cancer diagnoses can be attributed to HPV. Globally, the 2 most common vaccine-preventable HPV types are 16 and 18 which account for $70 \%$ of all cases. The next 6 most commonly-identified HPV types are 31, 33, 35, 45, 52 and 58 accounting for another $20 \%$ of the world's cervical cancer cases.

Papanicolaou (Pap) testing has facilitated earlier detection and treatment of cellular changes related to this oncogenic virus. In addition, HPV DNA self-testing kits are now available to women as a feasible alternative to Pap testing to increase screening for cervical cancer in populations

that may lack access to a health care provider or those who may avoid screening this due to embarrassment, discomfort, and anxiety. Because of the slow rate in which cervical cancer develops, it is a disease for which population screening is effective in identifying changes during the preinvasive phase when intervention can lead to a cure. However, approximately half of women diagnosed with HPV-related cancers continue to die.

\section{HPV Vaccine}

The current treatment of HPV-related cancers of physical removal of the lesion (cryotherapy, trichloracetic acid, laser, or surgical removal) may be ineffective. Recurrence is common due to the inability to totally remove the virus or the woman becoming re-infected after treatment. To more effectively combat this disease, the United States Food and Drug Administration (FDA) has approved 2 quadrivalent vaccines to prevent $\mathrm{HPV}$ infection: Gardasil ${ }^{\circledR}$ and Cervarix $^{\circledR}$. These medications have been deemed effective in eliminating the spread of HPV types 16 and 18 associated with the cancer etiology and HPV types 6 and 11 to which $90 \%$ of genital warts are attributed. These vaccines are currently approved for both females 9-26 years of age and males 9-21 years of age. Vaccination of boys is crucial to the health of women because of transmission via heterosexual contact.

In December 2014, the FDA announced the approval of Gardasil9 ${ }^{\circledR}$ (Human Papillomavirus 9-valent Vaccine, Recombinant). The new vaccine will cover $9 \mathrm{HPV}$ types, 5 
more HPV types than the previously marketed Gardasil. In addition to the four HPV types listed above, coverage will now include HPV types 31, 33, 45, 52 and 58 which will now have the potential to protect women against approximately $90 \%$ of cervical, vulvar, vaginal, anal and oropharyngeal cancers. Gardasil ${ }^{\circledR}$ was approved for use in females ages 9-26 and males ages 9-15 administered in 3 doses over 6 months. However, a 2-dose recommendation is being considered (3).

\section{Underutilization of the Vaccine}

While the HPV vaccines have shown to be effective in prevention of the spread of this disease, equitable, universal acceptance and use of the vaccine lags behind its recommended use. In the United States, only $37.6 \%$ of teenage girls and $13.9 \%$ of teenage boys received all 3 doses of the vaccine in 2013 . When initiated, the vaccination schedule of 3 doses is often not completed. While vaccination rates are slightly higher in some countries such as the United Kingdom, vaccination rates are much lower in the developing world. There is wide variation in vaccine use based on geographic and demographic variables. In the United States, vaccine initiation was lowest amongst non-Hispanic Black residents and persons with lower income as well as in rural areas with lack of access to a health care provider. There is an inverse correlation between vaccine initiation and cervical cancer incidence and mortality (4).

\section{Reasons for Underutilization}

The reasons for lack of wide-spread use are multifaceted and also vary by regions of the world. The infrastructure supporting the health care system within a country, gender disparities, financial concerns (both personal and nationwide), ethical and religious issues, lack of information, and individual preference have led to public debate of the policies that govern the administration of the HPV vaccine. Socio-economically marginalized girls and women worldwide experience disproportionately large burden of cervical cancer and face many barriers to HPV vaccination. These barriers include inability pay for health care services, decreased access to care, and a lack of community engagement to generate awareness about cervical cancer and early screening tools.

Politics has played a role in universal vaccination of all children against HPV. Health policies of a country and its political leaders have an impact on the introduction of a new pharmaceutical agent to a given population. The accessibility, manufacture, licensing, and distribution are facilitated or impeded policy adoption. Ideological features of the political environments and special interest groups may oppose or support policy proposals and alternatives. Concerns about safety and efficacy of HPV vaccines as well as the perception that pharmaceutical companies may be unduly influencing health care policy have key issues raised during policy debates. Originally, upon introduction of the vaccines in 2006, religious and conservative factions felt that its use would sanction teen sex and encourage promiscuity, but recent research refutes this claim.

Parents who have been polled regarding their support for the vaccine and/or their intent to vaccinate their own children have provided some insight into barriers that still need to be addressed. The most common response indicated that the health care did not recommend the vaccine (5). This suggests that education of health care providers is needed about the latest recommendations. Likewise, a lack of information on the part of the parent and the adolescent about the HPV is also a problem (6). As awareness is raised within the community regarding the value of vaccination, the health care consumer will be better informed and may be more proactive in requesting the vaccine from the provider. Compounding the problem, there is a lack of recognition of HPV as a sexually transmitted infection (STI) in the general population. Surveyed parents also believed that the HPV vaccine needed to be included in the national vaccine schedule and a reduction of cost would increase vaccination frequency. Sexual health dialogue between adolescents, their parents, and health care providers prior to first sexual encounter is an important aspect of care and often overlooked.

\section{Summary and Conclusions}

Cervical cancer is a major cause of mortality and morbidity to women worldwide. We have the pharmacologic means to prevent the suffering imposed by cervical and other HPV-related cancers through currently existing vaccines. While the cost for providing universal access to the vaccine might appear to be significant, fewer dollars will be spent in the long term by decreasing the incidence of cancer and other health conditions related to HPV (7). Accurate and detailed information about HPV, its consequences, and the vaccine to prevent it disseminated to all health care providers, policy makers, and the general public is essential for optimal health of our global society.

\section{Ethical Issues}

Not applicable.

\section{Conflict of Interests}

The author has no conflicts of interest to disclose.

\section{References}

1. Bruni L, Barrionuevo-Rosas L, Albero G, et al. Human Papillomavirus and Related Diseases in the World. Summary Report 2014. http://www.hpvcentre.net/ statistics/reports/XWX.pdf. Accessed January 24, 2015.

2. Rosales R, Rosales C. Immune therapy for human papillomaviruses-related cancers. World J Clin Oncol. 2014;5(5):1002-19. doi:10.5306/wjco.v5.i5.1002.

3. Food and Drug Administration (FDA). FDA News Release: FDA approves Gardasil 9 for prevention of 
certain cancers caused by five additional types of HPV, December 10, 2014. http://www.fda.gov/NewsEvents/ Newsroom/PressAnnouncements/ucm426485.htm. Accessed January 24, 2015. Published 2014.

4. Moss J, Reiter P, Brewer N. Correlates of human papillomavirus vaccine coverage: a state-level analysis. Sex Transm Dis. 2015;42(2):71-75. doi:10.1097/ OLQ.0000000000000225.

5. Donahue K, Stupiansky N, Alexander A, Zimet G. Acceptability of the human papillomavirus vaccine and reasons for non-vaccination among parents of adolescent sons. Vaccine. 2014;32(31):3883-3885. doi: 10.1016/j.vaccine.2014.05.035.

6. Firenze A, Marsala M, Bonanno V, et al. Facilitators and barriers HPV unvaccinated girls after 5 years of program implementation. Hum Vaccin Immunother. 2014;11(1):240-244. doi: 10.4161/hv.36158.

7. Fonseca A, de Lima Ferreira L. Systematic review of the cost-effectiveness of the vaccination against HPV in Brazil. Hum Vaccin Immunother. 2014;10(12):34843490. doi: 10.4161/hv.34410.

Copyright (c) 2015 The Author(s); This is an open-access article distributed under the terms of the Creative Commons Attribution License (http://creativecommons.org/licenses/by/4.0), which permits unrestricted use, distribution, and reproduction in any medium, provided the original work is properly cited. 